\section{Structure of weakly bonded PPG-silica nanocomposites}

\author{
Juliano A. Chakera, Karim Dahmouche ${ }^{\text {a }}$, Aldo F. Craievich ${ }^{\text {, }}$ \\ Celso V. Santilli ${ }^{\mathrm{a}}$ and Sandra H. Pulcinelli ${ }^{\mathrm{a}}$
}

\author{
${ }^{a}$ Institute of Chemistry, UNESP P.O. Box 355 14800-970, \\ Araraquara, SP, Brazil \\ ${ }^{b}$ Institute of Physics, USP P.O. Box 66318 05315-970, São Paulo, SP, \\ Brazil \\ Email:jachaker@iq.unesp.br
}

The structure of silica-polypropyleneglycol (PPG) nanocomposites with weak physical bonds between the organic (PPG) and inorganic (silica) phase, prepared by the sol-gel process, was investigated by small angle X-ray scattering (SAXS). These nanocomposite materials are transparent, flexible, have good chemical stability and exhibit high ionic conductivity when doped with lithium salt. Their structure was studied as a function of silica weight fraction $\mathrm{x}(0.06 \leq \mathrm{x} \leq 0.29)$ and $[\mathrm{O}] /[\mathrm{Li}]$ ratio (oxygens being of ether-type). The shape of the experimental SAXS curves agrees with that expected for scattering intensity produced by fractal aggregates sized between 30 and $90 \AA$. This result suggests that the structure of the studied hybrids consists of silica fractal aggregates embedded in a matrix of PPG. The correlation length of the fractal aggregates decreases and the fractal dimension increases for increasing silica content. The variations in structural parameters for increasing $\mathrm{Li}^{+}$doping indicate that lithium ions favor the growth of fractal silica aggregates without modifying their internal structure and promote the densification of the oligomeric PPG matrix.

\section{Introduction}

In recent years the study of organic-inorganic nanocomposites became a mushrooming field of investigation due to the promising applications of these materials in optics, electronics, mechanics, electrochemistry and biology (Beaucage et al., 1995). These hybrids are considered as biphasic materials, the organic and inorganic phases being mixed at a nanometric scale. The nature of the organic-inorganic interface has been used to classify these materials in two classes. In class I materials, organic and inorganic phases are linked together through weak bonds (hydrogen, van der Waals or ionic bonds), which give the cohesion to the whole structure. In class II, the two phases are linked through strong covalent bonds.

Small-angle X-ray scattering (SAXS) is a particularly adequate technique to study the structure of such hybrid materials because of their colloidal nature and high contrast in electronic densities between organic and inorganic phases (Rodrigues et al., 1992; Beaucage et al., 1995; Krakovský et al., 1998; Maene et al., 1998). The structure of class II siloxane-polyethyleneglycol (PEG) and siloxanepolypropyleneglycol (PPG) hybrids, which exhibit high transparency, flexibility, chemical stability and good ionic conductivity $\left(\sim 10^{-4} \mathrm{~S} / \mathrm{cm}\right)$ when doped with lithium salts, has recently been investigated by SAXS (Dahmouche et al., 1999a; Dahmouche et al., 1999b). The gelation and drying mechanisms of such systems were also studied using the same technique (Dahmouche et al., 1999c). These studies have clearly shown that the nature of the siloxane-polymer interface in class II materials determines their nanoheterogeneous structure. For these reasons, it appears interesting to investigate the structure of similar hybrids with only weak organic-inorganic bonds (class I).

In spite of their chemical stability being lower than class II nanocomposites, class I materials are of technological interest because of their high ionic conductivity (up to $\sim 10^{-3} \mathrm{~S} / \mathrm{cm}$ ) making them useful for application in electrochromic devices (Dahmouche et al., 1996; Dahmouche et al., 1998).

In this paper we report a SAXS study of PPG-silica class I nanocomposites prepared by the sol-gel process. A model of a fractal structure for the inorganic phase is proposed and the effects on the structure of silica content and lithium doping are described.

\section{Experimental}

PPG-silica hybrid materials were prepared by an ultrasonic assisted sol-gel method that promotes the homogeneity of insoluble reactants without adding a co-solvent. Tetraethoxysilane (TEOS) and hydrochloric acid aqueous solution $\left(0.1 \mathrm{~mol} . \mathrm{L}^{-1}\right)$ were stirred together under ultrasound (power 200watts) during $5 \mathrm{~min}$ to hydrolyze the TEOS $\left(\left[\mathrm{H}_{2} \mathrm{O}\right] /[\right.$ TEOS $\left.]=4\right)$ in acid conditions $(\mathrm{pH}=2.5)$. The desired quantities of $\mathrm{PPG}_{425}$ (425=molecular weight of PPG) having hydroxil therminal groups were added. Some of the samples were doped with lithium salt $\left(\mathrm{LiClO}_{4}\right)$. This salt was introduced and dissolved under ultrasound in order to obtain a transparent liquid. Then, the $\mathrm{pH}$ of the solution was increased up to 8 by adding $\mathrm{NH}_{4} \mathrm{OH}$. Gelation occurred within a few minutes. The gel samples were then allowed to dry under vacuum at $323 \mathrm{~K}$ for $48 \mathrm{~h}$.

Previous studies of silica-PEG hybrids prepared by a similar procedure demonstrated that only weak physical bonds are linking together the organic and inorganic phases (Judeinstein et al., 1994a; Judeinstein et al., 1994b; Wong, Bazin \& Sermon, 1997).

X-ray scattering measurements were performed using the SAXS beamline of the National Synchrotron Light Laboratory (LNLS), Campinas, Brazil. The SAXS beamline is equipped with an asymmetrically cut and bent silicon (111) monochromator which yields a monochromatic $(\lambda=1.608 \AA)$ and horizontally focused beam (Kellermann et al., 1997). A vertical position-sensitive X-ray gas detector and a multichannel analyzer were used to record the SAXS intensity, I(q), as a function of the modulus of the scattering vector $\mathrm{q}=(4 \pi / \lambda) \sin (\varepsilon / 2), \varepsilon$ being the scattering angle. Each SAXS spectrum corresponds to a data collection time of 300 s. Because of the small size of the incident beam cross-section at the detection plane, no mathematical desmearing of the experimental SAXS intensity function was needed.

The density of the hybrid samples were determined using a standard helium picnometer.

\section{SAXS intensity produced by fractal aggregates}

The scattering power $\mathrm{I}_{1}(\mathrm{q})$ of an isotropic aggregate consisting of spatially correlated particles is given by (Guinier, 1963)

$$
\mathrm{I}_{1}(\mathrm{q})=\phi \mathrm{P}(\mathrm{q}) \mathrm{S}(\mathrm{q})
$$

where $\phi$ is the number of primary particles per unit volume, $P(q)$ is the form factor of the particles and $S(q)$ is the structure factor that accounts for the spatial correlation between them.

The structure function for a set of correlated primary particles forming a fractal object is given by (Freltoft, Kjems \& Sinha,1986 and Teixeira, 1988)

$$
\begin{aligned}
S(q)= & \left(1+\left\{\left[1 /(q a)^{D}\right] \times\left\{[D \cdot \Gamma(D-1)] /\left[1+\left(1 / q^{2} \xi^{2}\right)\right]^{(D-1) / 2}\right\} \times\right.\right. \\
& \left.\left.\sin \left[(D-1) \tan ^{-1}(q \xi)\right]\right\}\right)
\end{aligned}
$$


where $a$ is the radius of the (assumed spherical) primary particles, $\xi$ is the correlation length or size parameter of the fractal structure, D is the fractal dimension and $\Gamma$ is the gamma function.

When the primary particles are very small $(\mathrm{a}<<\xi)$, the form factor $\mathrm{P}(\mathrm{q})$ is usually approximated by a constant value (Guinier, 1963):

$$
P(q)=\left(\rho_{p}-\rho_{m}\right)^{2} v^{2}
$$

where $\rho_{\mathrm{p}}$ is the electronic density of the (assumed homogenous) primary particles, $v$ their volume and $\rho_{\mathrm{m}}$ the electronic density of the medium where the particles are embedded.

Assuming that (i) the composite material is composed of $\mathrm{N}$ fractal aggregates per unit volume without spatial correlation between them, and (ii) each fractal aggregate consists of very small primary particles $(\mathrm{a}<<\xi)$ with volume $\mathrm{v}$ and electronic density contrast $\Delta \rho=\left(\rho_{p}-\rho_{m}\right)$, the SAXS intensity can be written as

$$
\mathrm{I}(\mathrm{q})=\mathrm{A} \mathrm{N}(\Delta \rho)^{2} \mathrm{v}^{2} \mathrm{~S}(\mathrm{q})
$$

where $\mathrm{S}(\mathrm{q})$ is given by equation (2) and $\mathrm{A}$ is a parameter which only depend on the scattering geometry and normalization procedure. Equation (4) becomes I(q) $\propto \mathrm{q}^{-\mathrm{D}}$ when $1 / \xi<<\mathrm{q}<<1 / a$.

At small $\mathrm{q}(\mathrm{q} \leq 1 / \xi)$, I(q) exhibits a Guinier-type behavior (Guinier, 1963). The radius of gyration of the isolated fractal aggregates, $\mathrm{Rg}$, can be determined by applying Guinier law. $\mathrm{Rg}$ is related to the correlation length $\xi$ by

$$
\operatorname{Rg}=[\mathrm{D}(\mathrm{D}+1) / 2]^{1 / 2} \xi
$$
1988):

The SAXS intensity extrapolated to $\mathrm{q}=0$ is given by (Teixeira,

$$
\mathrm{I}(0)=\mathrm{A} \mathrm{N}(\Delta \rho)^{2} \mathrm{v}^{2} \Gamma(\mathrm{D}+1)(\xi / \mathrm{a})^{\mathrm{D}}
$$

For $q$ values close to or larger than $1 / \mathrm{a}$, the constant value for the form factor of the primary particles (equation 3) is not an acceptable approximation. For $\mathrm{q} \gg \mathrm{1}$ /a the so-called Porod regime (Guinier, 1963 ) is attained and so the SAXS intensity is expected to exhibit an assymptotic behavior given by

$$
\mathrm{I}(\mathrm{q}) \propto 1 / \mathrm{q}^{4} \quad(\mathrm{q} \rightarrow \infty)
$$

\section{Results and discussion}

\subsection{Effect of silica content}

Fig. 1 shows double logarithmic plots of the experimental scattering intensity $\mathrm{I}(\mathrm{q})$ as a function of the modulus of the scattering vector, q, corresponding to $\mathrm{PPG}_{425}$-silica samples with different silica weight fraction $\mathrm{x}=\mathrm{m}\left(\mathrm{SiO}_{2}\right) /\left[\mathrm{m}\left(\mathrm{PPG}_{425}\right)+\mathrm{m}\left(\mathrm{SiO}_{2}\right)\right], \mathrm{m}\left(\mathrm{PPG}_{425}\right)$ and $\mathrm{m}\left(\mathrm{SiO}_{2}\right)$ being the weight of polymer and silica, respectively. Three regions can be distinguished in the scattering curves: i) a linear regime at medium $q\left(0.02 \AA^{-1}<q<0.22 \AA^{-1}\right)$, ii) a tendency to a constant intensity value at small $\mathrm{q}\left(\mathrm{q}<0.02 \AA^{-1}\right)$ and iii) an increase in the modulus of the slope at high $q$ values $\left(q>0.22 \AA^{-1}\right)$ which would correspond to the beginning of the Porod regime (equation 6). The fittings of the theoretical functions I(q) (equation 4) to experimental data are also shown in Fig.1. The fitted I(q) functions (continuous lines) are in good agreement with the experimental curves up to a value of $\mathrm{q}$ close to $1 / a$. This upper limit for good fitting is the expected consequence of using a constant value for the form factor of the primary particles. The parameter $a$, which corresponds to the size of the primary particles, was determined from the value $q_{c}$ corresponding to the crossover of the two linear ranges at large $q$ values $\left(a=1 / q_{c}\right)$.

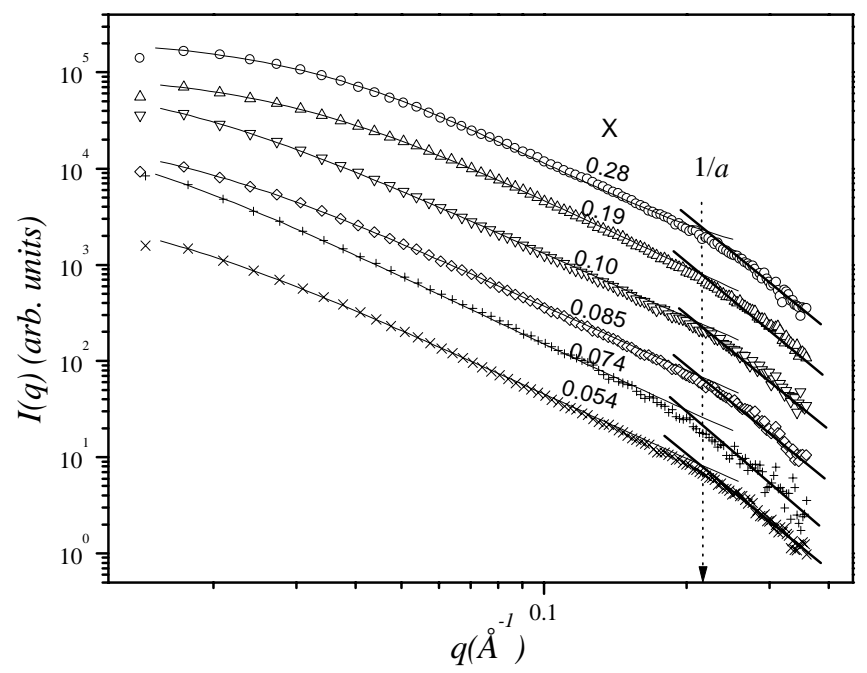

Fig. 1: Experimental SAXS curves corresponding to silica- $\mathrm{PPG}_{425}$ nanocomposites with different silica contents (x: silica weight fraction). The curves are vertically displaced for clarity. Continuous lines represent the results of fitting of equation (4) to experimental curves.

We have assumed that the X-ray scattering is due to the presence of inorganic (silica) fractal aggregates embedded in the PPG matrix. The aggregates are formed by clustering of primary particles of size $a$. The values of $a=1 / q_{c} \cong 5 \AA$ are the same for all samples, independently of their silica content. This implies that the volume $\mathrm{v}$ of the primary particles (equation 3 and 4) is independent of sample composition.

The correlation lengths $\xi$ and the fractal dimensions D of the clusters, the extrapolated $\mathrm{I}(0)$ values and the sample densities are plotted in Fig. 2 for different silica weight fractions, $x$. These values are the parameters corresponding to the best fittings of equation 2 to the different experimental.curves. The increase in sample density for increasing silica content, seen in Fig. 2, is expected because the values of the densities of pure PPG and bulk silica phase are equal to 1.0 and $2.2 \mathrm{~g} / \mathrm{cm}^{3}$, respectively.

The correlation length $\xi$ of the fractal silica aggregates decreases from 90 to $30 \AA$ for increasing silica content. This decrease in size can be simply explained as a consequence of steric effects. For diluted systems (low silica content) the number of nuclei for aggregate formation is small and so the fractal structure has an important available volume to be occupied by growth. On the contrary, more concentrated samples would contain a higher number of nuclei and so the growth of the fractal aggregates would stop after some eventual interpenetratation between neighbors. As a consequence of interactions between aggregates, the correlation length associated to each one becomes limited to values of the order of the average distance between them. Since the average distance is expected to decrease with silica content, the correlation length would also decrease.

The values of $\mathrm{I}(0)$ plotted in Fig. 2 exhibit an approximately linear increase for increasing silica weight fraction. Assuming $\Delta \rho$ independent of silica content and a constant $\mathrm{v}$ value, equation (5) predicts that the variation in $\mathrm{I}(0)$ only depends on $\mathrm{N}, \mathrm{D}$ and $\xi$. The observed increasing trend of $\mathrm{I}(0)$ was assigned to a predominant increasing effect of the number of aggregates $\mathrm{N}$ and fractal dimension $\mathrm{D}$, overcompensating the opposite effect expected from the variation in the correlation length $\xi$. 


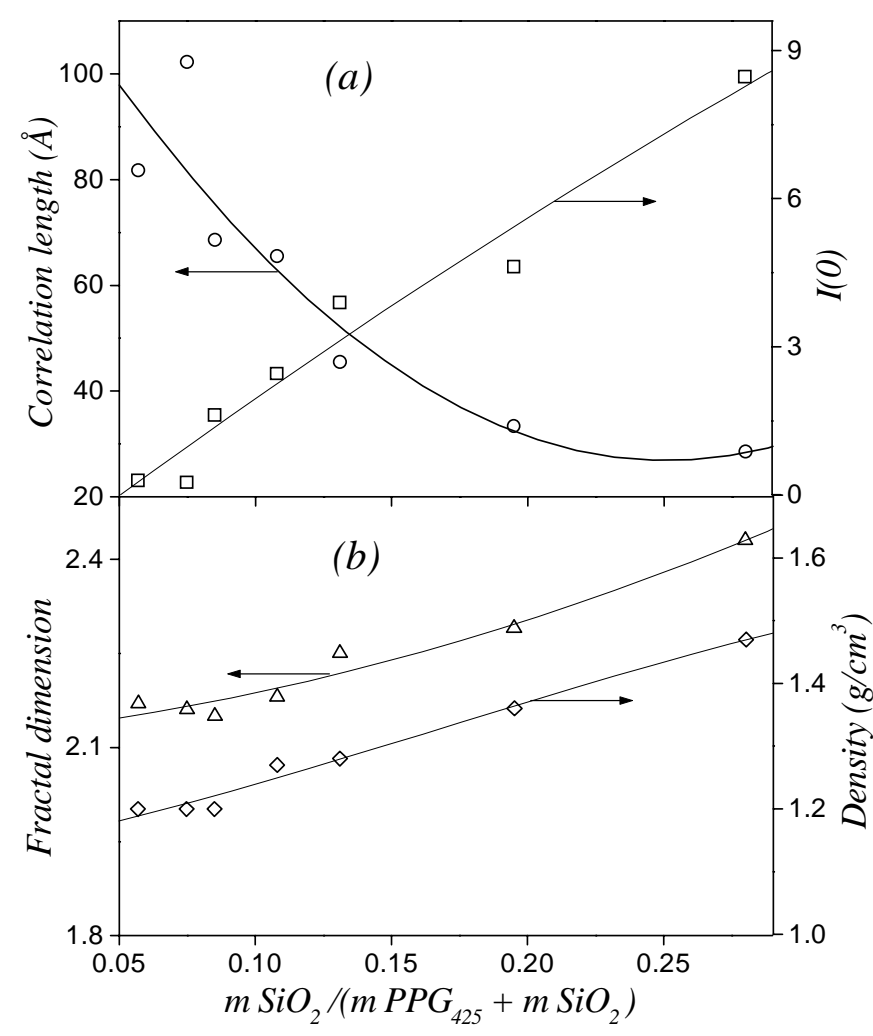

Fig. 2: (a) Correlation length of fractal aggregates and SAXS intensity extrapolated to $\mathrm{q}=0$ (arb. units), and (b) fractal dimension of the aggregates and sample density as functions of silica content.

A number of studies on aggregation of colloidal particles in liquid solvents have shown that fractal clusters are often formed. On the other hand computer simulation studies demonstrated that the fractal dimension of the clusters depends on the mechanism of aggregation (Meakin, 1989). The experimental value of the fractal dimension increases from 2.1 for low silica content $(\mathrm{x}=0.06)$ up to 2.5 for $\mathrm{x}=0.29$. The rather low $D$ values $(\mathrm{D} \cong 2.1)$ for low silica content suggest that the predominant mechanism is reaction-limited cluster-cluster aggregation (RLCCA) (Brown \& Ball, 1985). This is the mechanism that we expect for our synthesis procedure in which the first step involves acidcatalyzed hydrolysis and the second one the addition of a base (Brinker \& Scherer, 1990). For large silica content, the fractal dimension increases up to $\mathrm{D} \approx 2.5$. This value is expected for diffusion-limited particle-cluster aggregation (DLPCA) (Jullien. Kolb \& Botet, 1984). This growth process occurs through a condensation reaction of single monomers with growing clusters. Our results indicate that this mechanism is only predominant for high silica precursor content, i.e. when the specific number of reactant species can be a continuous source of monomers as required for particle-cluster aggregation.

\subsection{Effect of lithium content}

Fig. 3 exhibits double logarithmic plots of SAXS intensity as a function of the modulus of the scattering vector, I(q), for silica$\mathrm{PPG}_{425}$ samples containing different $[\mathrm{O}] /[\mathrm{Li}]$ ratios (the oxygen atoms are those of the ether type), all of them with the same weight fraction of silica, $x=0.14$. The SAXS spectra have a shape similar to those corresponding to non-doped composites, revealing that the fractal nature of silica aggregates is preserved when $\mathrm{Li}^{+}$ions are added.

The radius of the primary particles, $a=1 / q_{c}$, is equal to approximately $5 \AA$, irrespectively of lithium content. The values of the other parameters determined from the fitting of equation (4) to the experimental SAXS curves and the density of the material are plotted in Fig. 4 as functions of $[\mathrm{O}] /[\mathrm{Li}]$ ratio.

For low lithium content $([\mathrm{O}] /[\mathrm{Li}]=80)$, the density of the samples is approximately equal to the density of the undoped composite $(1.2$ $\mathrm{g} / \mathrm{cm}^{3}$ ). For increasing lithium content, up to $[\mathrm{O}] /[\mathrm{Li}]=15$, a slight increase in density is observed. As in a previous study of type II hybrids (Dahmouche et al., 1999b), this increase was assigned to the progressive filling of small empty spaces in the PPG matrix by adsorption of $\mathrm{Li}^{+}$in ether type oxygens.

For high lithium content, $[\mathrm{O}] /[\mathrm{Li}] \leq 15$, a pronounced increase in density is apparent. This behavior has already been observed in pure polyethers or hybrid silica-polyethers electrolytes (McCallum \& Vincent, 1987; Dahmouche et al., 1999b) and was attributed to the contraction of the matrix produced by the increase in degree of pseudo-crosslinking $\mathrm{O}-\mathrm{Li}^{+}-\mathrm{O}$ between PPG oligomers. We are calling pseudo-crosslinking the attractive ionic interactions between the oligomeric chains promoted by $\mathrm{Li}^{+}$addition, which lead to an overall effect of matrix contraction.

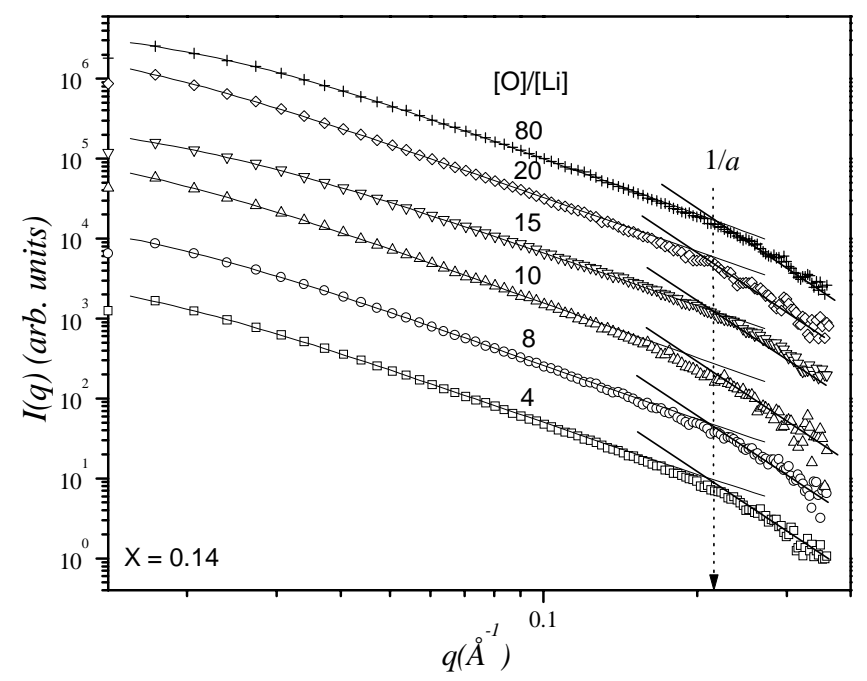

Fig. 3: Experimental SAXS curves corresponding to silica- $\mathrm{PPG}_{425}$ nanocomposites containing different $[\mathrm{O}] /[\mathrm{Li}]$ ratios. Continuous lines are the results of fittings of equation (2) to the experimental curves. The curves are vertically displaced for clarity. 


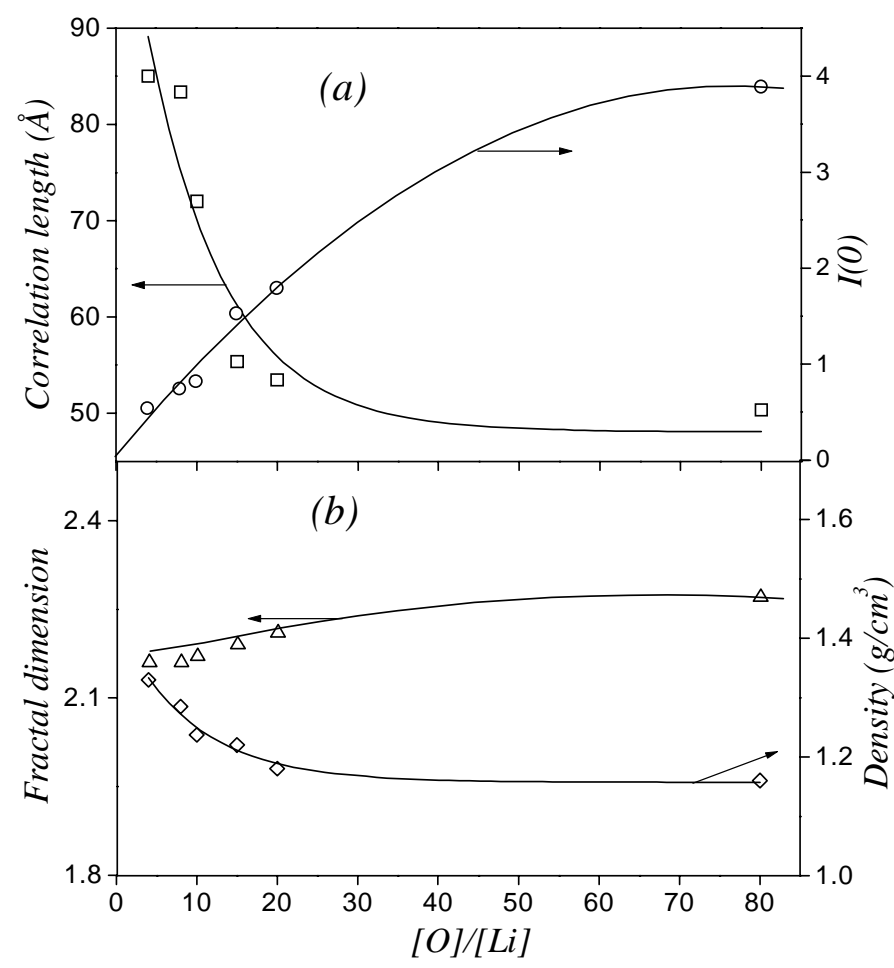

Fig. 4: (a) Correlation length of the fractal aggregates and SAXS intensity extrapolated to $\mathrm{q}=0$ (arb. units) and (b) fractal dimension of the aggregates and sample density as functions of $[\mathrm{O}] /[\mathrm{Li}]$.

Over the whole concentration range $(80 \leq[\mathrm{O}] /[\mathrm{Li}] \leq 4)$ the average correlation length, $\xi$, of the fractal silica clusters increases for increasing $\mathrm{Li}^{+}$doping while its fractal dimension $(\mathrm{D}=2.2)$ remains approximately constant. This reveals that lithium salt favors the growth of the fractal silica aggregates without appreciably changing their basic mechanism of formation. This effect associated to the electrolyte has already been observed in other sol-gel derived materials containing different salts (Brinker \& Scherer, 1990) and was associated to a decreasing effect of electrostatic repulsion between fractal clusters.

The decrease in extrapolated SAXS intensity, $\mathrm{I}(0)$, for increasing $\mathrm{Li}^{+}$doping indicates a predominant effect of the reduction in electron density contrast $\Delta \rho$. This effect is associated to an increase in the matrix density $\rho_{\mathrm{m}}$ due to i) the dissolution of $\mathrm{LiClO}_{4}$ and ii) the contraction promoted by pseudo crosslinking effects.

\section{Conclusion}

PPG-silica nanocomposites with weak organic-inorganic bonds were studied by SAXS for different silica contents. The fractal nature and the relevant structural characteristics of silica aggregates embedded in the PPG matrix were established. The size of the primary particles building up the fractal structure is invariant, the correlation length or parameter size of the aggregates decreases and the fractal dimension increases for increasing silica concentration.
Doping the PPG-silica composites with lithium salt does not noticeably affect the fractal nature of the silica aggregates and favors their growth. Lithium salt addition also promotes the formation of pseudo crosslinking effects between PPG oligomers in the matrix.

The authors acknowledge the collaboration of LNLS staff during SAXS experiments and financial support from FAPESP and PRONEX/MCT (Brazilian funding agencies).

\section{References}

Beaucage, G., Ulibarri, T. A., Black, E. P. \& Schaefer, D. W. (1995). Hybrid Organic-Inorganic Composites, ACS Symp. Series, 585, pp 97-110. Amer. Chem. Soc. Washington.

Brinker, C. J. \& Scherer, G. W. (1990). Sol-Gel Science, pp 203, Academic Press, San Diego.

Brown, W. D., \& Ball, C. D. (985) J. Phys. A. 18, L17-521.

Dahmouche, K., Atik, M., Mello, N. C., Bonagamba, T. J., Paneppucci, H., Aegerter, M. \& Judeinstein, P. (1996). Mat. Res. Soc. Symp. Proc, 435, 363-368.

Dahmouche, K., Santilli, C. V., Pulcinelli, S. H. \& Craievich, A. F. (1999a). J. Phys. Chem. B 103, 5775-5781.

Dahmouche, K., Santilli, C. V., Da Silva, M., Ribeiro, C.A., Pulcinelli, S. H. \& Craievich, A. F. (1999b). J .Non-Cryst. Solids, 248, 108113.

Dahmouche, K., Santilli, C. V., Chaker, J. A., Pulcinelli, S. H. \& Craievich, A. F. (1999c). Jap. J. Appl. Phys. (In press).

Dahmouche, K., De Souza P. H., Bonagamba, T. J., Paneppucci, H., Judeinstein, P., Pulcinelli, S. H. \& Santilli, C. V. (1998). J. SolGel Sci. Techn., 13, 909-913.

Guinier, A. (1963). X-ray Diffraction.Freeman and Company, San Francisco.

Judeinstein, P., Titman, J.,Stamm, M. \& Schmidt, H. (1994). Chem. Mater., 6, 127-134

Judeinstein, P., Brik, M. E., Bayle, J. P., Courtieu, J. \& Rault, J. (1994). M.R.S. Symp. Proc, 346, 937-942. MRS, Pittsburgh.

Jullien, R., Kolb, M. \& Botet, R. (1984) J. Phys. (Paris) 45, L211216.

Kellermann, G., Vicentin, F., Tamura, E., Rocha, M., Tolentino, H. Barbosa, A. F., Craievich, A. F. \& Torriani, I. L. (1997). J. Appl. Cryst. 30, 880-883.

Krakovský, I., Urakawa, H., Kajiwara, K. \& Kohjiya, S. (1998). J. Non-Cryst. Solids, 231, 31-40.

McCallum, J. R \& Vincent, A. C. (1987). Polymer Electrolytes Review. Elsevier, London.

Maene, N., Nair, B. N., D’Hooge, P., Nakao, S. I. \& Keizer, K. (1998). J. Sol-Gel Sci. Techn., 12, 117-134.

Meakin, P.(1989) The Fractal Approach to Heterogeneous Chemistry. D. Avnir ed. pp. 131-160. John Wiley and Sons.

Rodrigues, D. E., Brennan, A. B., Betrabet, C., Wang, B. \& Wilkes, G. L. (1992). Chem. Mater., 4, 1437-1446.

Wong, M.S.W., Bazin, N. \& Sermon, P.A (1997). J. Sol-Gel Sci. Techn., 8, 499-505. 